удК 332.1

Т. Д. Макаренко

Байкальский государственный университет, 2. Чита, Российская Федерация

Л. Б. Ковальчук

Байкальский государственный университет, 2. Чита, Российская Федерация

\title{
МЕТОДЫ И ПРИНЦИПЫ ДИАГНОСТИКИ СОЦИАЛЬНО-ЭКОНОМИЧЕСКИХ ПРОЦЕССОВ РЕГИОНА
}

\begin{abstract}
АННОТАЦИЯ. Идентификация методов диагностики трансформаций региональных социально-экономических процессов осуществлена в соответствии с иерархией целей системной диагностики региональных процессов. Исследованы методы, используемые в существующих системах диагностики. Аргументирована необходимость их адаптации и корректировки для диагностики трансформаций региональных процессов. Корректировка предлагаемого методического инструментария осуществлена в направлении использования математического аппарата. Предложено, в целях адаптации существующего методического инструментария, использовать теорию нечеткой логики. Обосновано, что использование данной теории позволит расширить возможности формирования надежной информационной базы диагностики. Показано, что достижение целей системной диагностики трансформаций обусловливает необходимость интеграции методов естественных, математических и гуманитарных наук. Использование предложенного методического инструментария диагностики трансформаций социально-экономических процессов поставлено в зависимость от ее принципиальных основ. Предложена совокупность устойчивых организующих принципов процесса диагностики региональной социально-экономической системы. Сформированная принципиальная основа диагностики трансформаций объединяет общенаучные и синергетические принципы. Обосновано, что интеграция указанных принципов обеспечит возможность многоаспектной и многофакторной диагностики регионального развития. Показано, что предложенные методы и принципы непротиворечиво дополняют друг друга и выступают важнейшими компонентами методологической основы диагностики трансформаций региональной социально-экономической системы.

КЛЮЧЕВЫЕ СЛОВА. Трансформации региональных социально-экономических процессов; системная диагностика; методический инструментарий; принципиальные основы.

ИНФОРМАЦИЯ О СТАТЬЕ. Дата поступления 25 ноября 2016 г.; дата принятия К печати 11 января 2017 г.; дата онлайн-размещения 31 марта 2017 г.
\end{abstract}

T. D. Makarenko Baikal State University, Chita, Russian Federation

L. B. Kovalchuk Baikal State University, Chita, Russian Federation

\section{METHODS AND PRINCIPLES OF DIAGNOSING REGIONAL SOCIO-ECONOMIC PROCESSES}

ABSTRACT. Identifying methods of diagnosing transformations of regional socio-economic processes is carried out in accordance with the target hierarchy for the system diagnosis of regional processes. The article investigates the methods used in the existing diagnostic systems. It argues the necessity of their adaptation and adjustment of the diagnostic methods of regional transformation processes. It makes the

(C) Т. Д. Макаренко, Л. Б. Ковальчук, 2017

\section{Baikal Research Journal}


correction of the suggested methodical instrumentarium in the direction of using a mathematical apparatus technique. In order to adapt the existing mathematical apparatus technique, it offers to use the theory of fuzzy logics and substantiates that using this theory will allow to expand the possibilities of building a secure data base of diagnosis. It is shown that achieving the objectives of system diagnosis of transformations determines the necessity of integrating methods of natural, mathematical and humanitarian sciences. The use of the suggested mathematical apparatus technique for diagnosing transformation of socio-economic processes is made dependent on principle basics. The article offers an aggregate of sustained organizing principles of the diagnostic process for the regional socio-economic system. The formulated principal basis of diagnosing transformations integrates general scientific and synergetic principles. It is substantiated that integration of the these principles will provide an opportunity of multi-aspect and multi-factor diagnosis of the regional development. It is shown that the suggested methods and principles complement each other consistently and act as the most important components of the methodological basis of diagnosing transformations of the regional socio-economic system.

KEYWORDS. Transformation of regional socio-economic processes; system diagnosis; methodological mathematical apparatus technique; principled foundations.

ARTICLE INFO. Received November 25, 2016; accepted January 11, 2017; available online March 31, 2017.

Диагностика состояния и характера трансформаций социально-экономических процессов региона является сложной, многоаспектной задачей, требующей использования разнообразного набора методов исследования. Необходимым условием при этом является соответствие выбранного метода определенной цели системной диагностики, чтобы этот метод обеспечивал возможность выявления трансформаций процессов социально-экономического развития региона.

Первоначальной целью диагностики является выявление наличия и идентификации характера трансформаций региональных социально-экономических процессов [1]. Одним из методов реализаций данной цели может выступать сопоставительный (сравнительный) анализ. Убедительным аргументом в пользу приведенного предположения выступает широкое использование указанного подхода в разработанных диагностических системах. [2, с. 17-29]. Так, исследователи О. Г. Дмитриева, О. В. Кузнецова, А. В. Кузнецов, В. Н. Лексин на основе сравнительного анализа диагностировали общее состояние развития региона, отдельные его сферы (экономическая, социальная), выявили проблемные ситуации. Одно из преимуществ названного метода состоит в возможности его использования при оценках неограниченного числа показателей, отражающих результативность социально-экономических процессов региона. Указанные показатели сравниваются с аналогичными, интерпретируемыми в качестве нормы. Следует отметить, что обоснование уровня нормативных показателей является весьма сложным процессом. Применительно к региональной диагностике в качестве нормы могут выступать показатели, характеризующие среднероссийский уровень результативности социально-экономических процессов.

С учетом целей диагностики наиболее важным является выявление характера, трансформаций и исследование их динамики в разрезе структуры региональных социально-экономических процессов.

При формировании информационной основы диагностики необходимо ориентироваться на систему ее целей с тем, чтобы совокупность показателей позволяла дать оценку результативности достижения этих целей. Следует заметить, что указанную совокупность необходимо откорректировать с учетом наличия информационной основы для расчета соответствующих нормативных показателей, в данном случае среднероссийских. Необходимо указать, что названное обстоятельство следует при-

\section{Baikal Research Journal}


знать одним из недостатков рассматриваемого метода: вне диагностики могут оказаться значимые, но не имеющие нормативной основы для сравнения показатели.

Нивелировать в определенной степени указанный недостаток возможно посредством расширения возможностей метода сравнительного анализа через включение в аналитическую базу минимальных и максимальных показателей, характеризующих результативность социально-экономических процессов региона за определенный исторический период, например 5 лет [3, с. 75-79]. При этом появляется необходимость обоснования шкалы оценки, позволяющей формировать качественные характеристики выявленных трансформаций, например: небольшие; значимые; глубокие. Непременным условием при этом является установление характера данных трансформаций: положительные или отрицательные.

Полученные результаты выступают основой осуществления, локализации и идентификации трансформаций исследуемых процессов региона. Необходимым условием реализации данной цели является выявление взаимосвязей между исследуемыми процессами. Наиболее приемлемым методом решения поставленной задачи, на наш взгляд, выступает моделирование. С позиции диагностики сформированная модель позволит воспроизвести наиболее существенные связи между процессами региональной социально-экономической системы, установить функциональную зависимость, формализовать ее посредством математических моделей. В свою очередь использование математических и логических моделей обеспечит возможность воспроизводства нескольких сценариев, включающих механизмы оптимизации отрицательных и положительных трансформаций данных процессов. Таким образом, названный метод позволяет не только диагностировать состояние развития региона, но и обеспечивает основу прогнозирования результативности социально-экономических процессов.

В дальнейшем процессы диагностики осуществляются в направлении выявления наиболее существенных трансформаций в социальной или экономической сферах. На данном этапе сопоставляются входы и выходы в региональную социально-экономическую систему. Эту процедуру в достаточно полном объеме обеспечивает балансовый метод. Его использование позволяет генерировать необходимую информацию для определения спектра альтернативных траекторий социально-экономических процессов в увязке с ресурсным потенциалом региона.

С целью типологизации исследуемых процессов для их последующего ранжирования по типам трансформаций, считаем необходимым использование метода кластеризации. Типологизация обеспечит информационную основу разработки специализированных блоков мер в области социально-экономического развития региона. На основании полученной информации становится возможной оценка глубины трансформаций в соответствии с их типами (экономические, социальные) и видами (положительные, отрицательные). Решение данной задачи возможно посредством метода экспертных оценок. При этом необходимым условием его применения является формирование параметров и показателей, характеризующих трансформации изучаемых процессов. В силу многофункциональности социально-экономической системы региона основой данных показателей может служить многообразие соответствующих измерителей. Данное обстоятельство и обусловливает использование указанного метода, позволяющего свести разного рода показатели к одному - интегральному. Наличие системы показателей оценки трансформаций обеспечит количественную оценку взаимовлияния типов исследуемых процессов. Для установления типа связей и глубины взаимовлияния социально-экономических процессов рекомендуется использовать метод регрессионного анализа, результаты которого позволят сгруппировать исследуемые процессы по признакам глубины и вида трансформаций [4].

\section{Baikal Research Journal}

электронный научный журнал Байкальского государственного университета 
С методической точки зрения можно констатировать, что использование совокупности представленных методов обеспечит достижение целей диагностики трансформаций региональных социально-экономических процессов (табл.).

\section{Цели и летоды диагностики трансфорлаций социально-эконолических процессов региона}

\begin{tabular}{|l|l|}
\hline \multicolumn{1}{|c|}{ Содержание целей диагностики } & \multicolumn{1}{|c|}{ Метод диагностики } \\
\hline $\begin{array}{l}\text { Выявление трансформаций социально-экономических процессов } \\
\text { региона, идентификация их характера }\end{array}$ & Сопоставительный анализ \\
\hline $\begin{array}{l}\text { Локализация трансформаций региональных социально-экономи- } \\
\text { ческих процессов }\end{array}$ & Моделирование \\
\hline $\begin{array}{l}\text { Оценка симметричности трансформаций в экономике и социаль- } \\
\text { ной сфере региона }\end{array}$ & Балансовый \\
\hline $\begin{array}{l}\text { Типологизация трансформаций социально-экономических про- } \\
\text { цессов региона }\end{array}$ & Кластеризация \\
$\begin{array}{l}\text { Оценка трансформаций региональных социально социально-эко-- } \\
\text { номических процессов }\end{array}$ & Экспертные оценки \\
\hline $\begin{array}{l}\text { Оценка взаимосвязей между трансформациями социально-эконо- } \\
\text { мических процессов региона }\end{array}$ & Регрессионный анализ \\
\hline $\begin{array}{l}\text { Группировка региональных социально-экономических процессов } \\
\text { по типам и видам трансформаций }\end{array}$ & $\begin{array}{l}\text { Теоретико-графовое моде- } \\
\text { лирование }\end{array}$ \\
\hline
\end{tabular}

Представленный в таблице методический инструментарий обеспечивает возможность применения современных информационных технологий, формирование надежной информационной базы [5, с. 37-56]. Центральное место в предлагаемой методической совокупности занимают методы математического моделирования, основанные на использовании теории нечеткой логики, они обеспечивают возможность получения и преобразования качественных показателей регионального развития, определяют функциональные зависимости между различными элементами и уровнями диагностируемой системы. Особенностью данного инструментария является интегрированное использование методов естественных, математических и гуманитарных наук, в частности методов сравнительного и регрессионного анализа совместно с когерентным; математических моделей совместно с аналитическими; лингвистические переменные, используемые экспертами, совместно со статистическими данными и т. п.

Эффективность и результативность практического использования данного комплекса находятся в зависимости от принципиальных основ построения и функционирования предлагаемой системы диагностики, установления четких правил их применения.

Принимая во внимание многоаспектность и многофакторность социально-экономического развития региона, считаем необходимым сформировать совокупность принципов, руководство которыми обеспечит диагностике необходимый уровень точности в процессе использования представленного методического инструментария.

В рамках указанных методических подходов необходимо, в первую очередь, опираться на общенаучные принципы: адекватность, достоверность, корректность, динамичность, многомерность, комплексность, сопоставимость, интегративность [6, с. 44-48].

Руководство принципом адекватности требует формирования такой системы диагностики, которая обеспечивает выявление трансформаций каждого процесса, который выделен в социально-экономической системе, в качестве самостоятельного элемента. Другими словами, данный принцип обеспечивает наличие методического инструмента для диагностики любого процесса указанной системы на предмет трансформаций [7, с. 767-774].

\section{Baikal Research Journal}


Принцип достоверности обусловливает необходимые требования к совокупности показателей, характеризующих трансформации исследуемых процессов. Это касается общего числа и соотношения объективных и субъективных показателей. Очевидным необходимо признать преобладание в данной совокупности количественных показателей, значения которых получены формализованными методами, качественные же, основанные на субъективных оценках, в соответствии с принципом многомерности должны выступать как дополнительная информация, способствующая повышению точности диагностики [8, с. 493-501].

Важным принципом процесса диагностики является корректность, которая предполагает обоснованность используемых методов, выводов, гипотез. Соблюдение этого принципа обусловливает использование в процессе диагностики проверенных достоверных показателей.

В той связи, что диагностике подвергается система процессного типа, необходимым условием становится соблюдение принципа динамичности. В данном случае основой его исполнения является выявление трансформаций во временном отрезке, через исследование факторов, их возникновения и развития [9, с. 27-43].

Процедура обоснования параметров, показателей, отражающих трансформации социально-экономических процессов базируется на принципе "многомерность». Данное обстоятельство обусловлено необходимостью учета при диагностике множества аспектов экономического и социального типа, которые не имеют единого измерителя [10, с. 60-65].

Принцип комплексности предполагает при оценке трансформаций учитывать факторы, относящиеся не только к видам экономической деятельности, но и к отраслям социальной сферы. В процессе классификации показателей формируются группы, комплексно отражающие трансформации, например, в образовании, жилищно-коммунальном комплексе и т. д. [11, с. 64-87]. По данным группам формируются обобщающие показатели, которые трансформируются при соблюдении принципа «интегративность», в интегральный показатель, характеризующий трансформацию социально-экономической системы региона в целом [12, с. 307-316].

Рассмотренные методы и принципы непротиворечиво дополняют друг друга и выступают важнейшими компонентами методологической основы диагностики региональной социально-экономической системы.

\section{Список использованной литературы}

1. Бобрышев А. Н. К вопросу о детерминантах и поиске новых форм регионального развития [Электронный ресурс] / А. Н. Бобрышев, М. Ю. Казаков // Управление экономическими системами : электронный научный журнал. - 2011. - № 9. - Режим доступа: http://uecs.ru/uecs-33-332011/item/608-2011-09-13-06-52-21.

2. Герасимов А. Н. Инструментарий диагностики региональных проблем и перспектив социально-экономического развития / А. Н. Герасимов, Е. Н. Громов, Е. Г. Дьяченко // Экономический анализ: теория и практика. - 2016. - № 3 (450). - С. 17-29.

3. Таранова И. В. Диагностика параметров социально-экономического и пространственного развития регионов / И. В. Таранова // Научные труды SWorld. $-2013 .-$ T. 32, № 1. - С. $75-79$.

4. Дованков А. Ю. Методика оценки социально-экономического административно-территориального образования субъекта Российской Федерации / А. Ю. Дованкова // Региональная стратегия устойчивого социально-экономического роста : Межрегион. науч.-практ. конф. : тез. докл. - Екатеринбург : Урал. отд-ние РАН, 1995. - Ч. 2.

5. Когут А. Е. Информационные основы регионального социально-экономического мониторинга : монография / А. Е. Когут, В. Е. Рохчин. - СПб., 1995. - 143 с.

6. Харченко Е. В. Управление региональным хозяйственным комплексом в условиях структурно-динамической нестабильности / Е. В. Харченко // Известия Иркутской государственной экономической академии. - 2011. - № 1 (75). - С. 44-48.

\section{Baikal Research Journal}


7. Чупров С. В. Особенности управления инновационной реиндустриализацией в нестационарной среде региональной экономики / С. В. Чупров // Известия Иркутской государственной экономической академии. - 2015. — T. 25, № 5. - C. 767-774. - DOI: 10.17150/1993-3541.2015.25(5).767-774.

8. Баенхаева А. В. Агрегированный критерий оценки качества высших учебных заведений Российской Федерации / А. В. Баенхаева, М. П. Базилевский, С. И. Носков // Известия Иркутской государственной экономической академии. - 2016. - Т. 26, № 3. C. 493-501. - DOI: 10.17150/1993-3541.2016.26(3).493-501.

9. Пространственное развитие региона: оценка, проблемы, перспективы : монография / Е. В. Бережная, А. Н. Бобрышев, М. Ю. Казаков, А. А. Мирохина, О. В. Бережная. - М. : Буки Веди. - 2012. - 212 с.

10. Сценарное моделирование в управлении региональным развитием / Т. С. Клебанова, Л. С. Гурьянова, Т. Н. Трунова, А. Ю. Смирнова // Бизнес информ. - 2012. № 10 . - C. $60-65$.

11. Лексин В. В. Региональная диагностика: сущность, предмет и метод, специфика применения в современной России / В. В. Лексин // Российский экономический журнал. - 2003. - № 9-10. - С. 64-87.

12. Суспицын С. А. Оценки потенциального экономического роста регионов России на основе региональных фенотипов / С. А. Суспицын // Регион: экономика и социология. 2010. - № 4. - C. 307-316.

\section{References}

1. Bobrishev A. N Kazakov M. Yu. On issue of determinants and search for new forms of regional development. Upravlenie ekonomicheskimi sistemami: elektronnyi nauchnyi zhurnal = Management of Economic Systems: Scientific Electronic Journal, 2011, no 9. Available at: http://uecs.ru/uecs-33-332011/item/608-2011-09-13-06-52-21. (In Russian).

2. Gerasimov A. N., Gromov E. I., Tkachenko E. G. Instrumentarium of diagnosing regional problems and prospects of socio-economic development. Ekonomicheskii analiz: teoriya $i$ praktika = Economic Analysis: Theory and Practice, 2016, no. 3, pp. 17-29. (In Russian).

3. Taranov I. V. Diagnosing parameters of socio-economic and spatial development of regions. Nauchnye trudy SWorld. = Transactions SWorld, 2013, vol. 32, no. 1, pp. 75-79. (In Russian).

4. Dovankov A. Yu. Methods of assessing socio-economic administrative and territorial formation of the Russian Federation entity. Regional'naya strategiya ustoichivogo sotsial'no-ekonomicheskogo rosta: Mezhregional'naya nauchno-prakticheskaya konferentsiya [Regional strategy of sustainable socio-economic growth. Materials of Inter-Regional Research Conference]. Yekaterinburg, Ural Branch of Russian Academy of Sciences Publ., 1995. Pr. 2. (In Russian).

5. Kogan A. E., Rohchin V. E. Informatsionnye osnovy regional'nogo sotsial'no-ekonomicheskogo monitoringa [Information basics of regional socio-economic monitoring]. Saint Petersburg, 1995. $143 \mathrm{p}$.

6. Kharchenko E. V. Regional economic complex management in terms of structural and dynamic instability. Izvestiya Irkutskoi gosudarstvennoi ekonomicheskoi akademii = Bulletin of Irkutsk State Economics Academy, 2011, no. 1 (75), pp. 44-48. (In Russian).

7. Chuprov S. V. Features of innovative reindustrialization management in non-stationary environment of regional economy. Izvestiya Irkutskoi gosudarstvennoi ekonomicheskoi akademii $=$ Bulletin of Irkutsk State Economics Academy, 2015, vol. 25, no. 5, pp. 767-774. DOI: 10.17150/1993-3541.2015.25(5).767-774. (In Russian).

8. Bayenkhayeva A. V., Bazilevsky M. P., Noskov S. I. Aggregated criterion for evaluating higher education institutions in the Russian Federation. Izvestiya Irkutskoi gosudarstvennoi ekonomicheskoi akademii = Bulletin of Irkutsk State Economics Academy, 2016, vol. 26, no. 3, pp. 493-501. DOI: 10.17150/1993-3541.2016.26(3).493-501. (In Russian).

9. Berezhnaya E. V., Bobrishev A. N., Kazakov M. Yu, Mirohina A. A., Berezhnaya O. V. Prostranstvennoe razvitie regiona: otsenka, problemy, perspektivy [Spatial regional development: assessment, problems, prospects]. Moscow, Buki Vedi Publ., 2012. 212 p.

10. Klebanov T. S., Guryanov L. S., Trunov T. N, Smirnova A. Y. Scenery modeling in regional development management. Business Inform, 2012, vol. 10, pp. 60-65. (In Russian).

\section{Baikal Research Journal}


11. Lexin V. V. Regional diagnosis: essence, subject and method, specificity of use in modern Russia. Rossiiskii ekonomicheskii zhurnal = Russian Economic Journal, 2003, vol. 9-10, pp. 64-87. (In Russian).

12. Suspitzin S. A. Assessments of potential economic growth of Russia's regions on the basis of regional phetypes. Region: ekonomika $i$ sotsiologiya = Region: Economics and Sociology, 2010, vol. 4, pp. 307-316. (In Russian).

\section{Информация об авторах}

Макаренко Талара Длитриевна - доктор экономических наук, профессор, директор, Читинский институт, Байкальский государственный университет, 672000, г. Чита, ул. Анохина, 56, e-mail: academy@economy.chita, mail@narhoz-chita.ru.

Ковальчук Людлила Борисовна - кандидат экономических наук, доцент, заместитель директора по научной и воспитательной работе, Читинский институт, Байкальский государственный университет, 672000, г. Чита, ул. Анохина, 56, e-mail: kovalchuklb@mail.ru.

\section{Authors}

Tamara D. Makarenko - Doctor habil. (Economics), Professor, Director, Chita Institute, Baikal Sate University, 56 Anokhin St., 672000, Chita, Russian Federation; e-mail: academy@economy.chita,mail@narhoz-chita.ru.

Lyudmila B. Kovalchuk - PhD in Economics, Associate Professor, Deputy Director for Scientific and Educational Work, Chita Institute, Baikal Sate University, 56 Anokhin St., 672000, Chita, Russian Federation; e-mail: kovalchuklb@mail.ru.

\section{Библиографическое описание статьи}

Макаренко Т. Д. Методы и принципы диагностики социально-экономических процессов региона / Т. Д. Макаренко, Л. Б. Ковальчук // Baikal Research Journal. — 2017. T. 8, № 1. - DOI: 10.17150/2411-6262.2017.8(1).8.

\section{Reference to article}

Makarenko T. D., Kovalchuk L. B. Methods and principles of diagnosing regional socio-economic processes. Baikal Research Journal, 2017, vol. 8, no. 1. DOI: 10.17150/24116262.2017.8(1).8. (In Russian).

\section{Baikal Research Journal}

